\title{
Applications and analytical tools of cell communication based on ligand-receptor interactions at single cell level
}

Fen Ma ${ }^{1,2}$, Siwei Zhang ${ }^{1,2}$, Lianhao Song ${ }^{1,2}$, Bozhi Wang ${ }^{1,2}$, Lanlan Wei ${ }^{1,2,3^{*}}$ and Fengmin Zhang ${ }^{1,2^{*}}$ (1)

\begin{abstract}
Background: Cellular communication is an essential feature of multicellular organisms. Binding of ligands to their homologous receptors, which activate specific cell signaling pathways, is a basic type of cellular communication and intimately linked to many degeneration processes leading to diseases.

Main body: This study reviewed the history of ligand-receptor and presents the databases which store ligand-receptor pairs. The recently applications and research tools of ligand-receptor interactions for cell communication at single cell level by using single cell RNA sequencing have been sorted out.

Conclusion: The summary of the advantages and disadvantages of analysis tools will greatly help researchers analyze cell communication at the single cell level. Learning cell communication based on ligand-receptor interactions by single cell RNA sequencing gives way to developing new target drugs and personalizing treatment.
\end{abstract}

Keywords: Cell communication, Ligand-receptor interactions, Single cell RNA sequencing, Target therapy, Tumor microenvironment

\section{Background}

Every cell in multicellular organisms lives in a variety of signaling environments, and its biological behavior is regulated by extracellular signaling molecules. Cell communication is a basic feature of multicellular organisms [1]. The dynamic communicating network formed through communication and cooperation between cells plays crucial roles in numerous biological processes [2, 3]. By specifically recognizing and binding with signal molecules, receptors converting these molecules into intracellular signals and perform specific physiological functions. Complex cellular reactions begin with the binding of ligands to their homologous receptors, which activate specific cell signaling pathways [4]. Therefore,

\footnotetext{
*Correspondence: 2743623823@qq.com; fengminzhang@ems.hrbmu.edu.cn ${ }^{1}$ Department of Microbiology, Harbin Medical University, Harbin 150081, China

Full list of author information is available at the end of the article
}

the analysis of ligand-receptor pairs interactions are the basis for understanding cell behavior and responses to neighboring cells.

Recently, Single-cell RNA sequencing (scRNA-seq) has led to breakthroughs in scientific research. scRNA-seq conducted analysis on cellular basis, which makes it feasible to investigate undiscovered cellular commutations. There are some progresses that have been learning intercellular communication through scRNA-seq, which continues to advance at such a rapid pace that even recent reviews $[3,5,6]$. Many research has focued on ligandreceptor interaction-based strategy to construct cellular communication network, rather than the physically vicinal structure-based strategy [3]. Ligand-receptor interactions are effective way to learn cellular communication at single cell level.

Here, we reviewed the recently research progress of ligand-receptor interaction for intercellular communication through scRNA-seq under multiple conditions, and 
compared the analysis tools for ligand-receptor interactions on single-cell level. These will help to better understand the crucial role of ligand-receptor interactions in cell communication research.

\section{Ligand-receptor pairs in disease}

In 1971, the receptor was first reported to play an important role in cancer with estrogen receptor expressed dysregulated in breast cancer patients [7]. Later, Obesity and hyperinsulinemia were later found to be caused by insulin receptor deficiency in mice [8]. The relationship between ligand-receptor and disease has been discovered gradually. Ligands and receptors are involved in disease development mainly in two ways: structural or genetic alterations and the expression of receptor/ligand change. Familial Hypercholesterolemia (FH), which is caused by low density lipoprotein receptor (LDLR) deficiency [9], and diabetes mellitus which is a consequence of abnormal insulin receptor.

Immune checkpoint is a common and widely studied receptor ligand in tumor research. They are ligandreceptor pairs that inhibit the interaction of the immune response. Cytotoxic $\mathrm{T}$ lymphocyte-associated antigen- 4 (CTLA-4) was the first immune checkpoint receptor identified. Studies have shown that CTLA-4 is closely related to tumor progression and treatment, and blocking the inhibitory effect of CTLA-4 can enhance the effective immune response against tumor cells [10]. Subsequently, researchers found that various tumor cells can inhibit the function of $\mathrm{T}$ cells by working on the immune checkpoint programmed death 1 (PD-1), which allows the tumor cells to escape from immune surveillance $[11,12]$. Moreover, the expression of immune checkpoints, such as CD137 (4-1BB), inducible co-stimulator (ICOS), T cell immunoglobulin and mucin domain 3 (TIM-3) changed in the tumor microenvironment could affect tumor progression [13]. The understanding of ligand-receptor interaction is the foundation for current studies of intercellular communication. It gives researchers a deeper insight into the processes of cellular biological activity and disease progression.

With the increasing discovery on receptors and ligands and their interactions, compilation by sorting and summarizing relevant information into ligand-receptor databases has been done continuously to facilitate research (Table 1). Although these databases comprehensively organize the available information on ligand-receptor interaction, there are still undiscovered receptors, ligands and their relationships. Therefore, after analyzing the existing ligand-receptor complexes, researchers developed simulation analysis software for the prediction of ligand-receptor interactions, for example, DOCK [14], Autodock [15, 16], AutoDock Vina, iGEMDOCK, and
RosettaDock [17]. Numerous inductive databases and simulation tools help researchers to better study ligandreceptor complexes and their interactions, which in turn contributes to drug development and disease treatment.

\section{scRNA sequencing}

With the development of research, the researchers found that different types of cells in the same sample (such as tissue, blood) have different function. Studies have shown that research methods which measure characteristics only on population level may average or dilute important differences between cells. Due to the lack of synchronization among cells, stochastic events of protein production are difficult to observe directly with measurements on large ensembles of cells $[48,49]$. Sequencing technology has developed from the first generation to the third generation based on nanopore and single molecule real-time sequencing $[50,51]$, which only improves the depth, accuracy and throughput of sequencing. However, none of them can re-establish cellular barriers and analyze gene expression at single cell level. Such shortcoming poses a problem for the study of tissue, blood, and other experimental samples consisting of mix multiple cells types. To address such problem, single cell sequencing have been developed to perform high-throughput sequencing of the genome, transcriptome, epigenome, etc. at single cell level [52].

Among them, scRNA-seq was first reported in 2009 [53] by separating single oocytes in Eppendorf tubes containing a lysis buffer [54]. scRNA-seq have enabled the simultaneous classification of thousands of cells in a single assay based on transcriptome profiling [53], which means several novel or rare cell-types that have opportunities to be discovered. The Human Cell Atlas [55] and $\mathrm{NIH}$ Brain Initiative projects [56] intend to sequence all cell types present in the human body and brain, respectively. Single-cell transcriptomic atlases provide unprecedented resolution to reveal complex cellular events and deepen our understanding of biological systems [57]. More importantly, the advances of scRNA-seq provide the possibilities to investigate undiscovered cellular commutations (Fig. 1).

\section{Applications of scRNA sequencing in receptor-ligand analysis}

The scRNA sequencing has been applied in various research fields to learn the important roles of ligandreceptor interactions in such cellular communications (Fig. 2).

\section{Disease research}

Currently, malignant tumors are one of the main threats to human life. Zemin Zhang et al. found that dendritic 


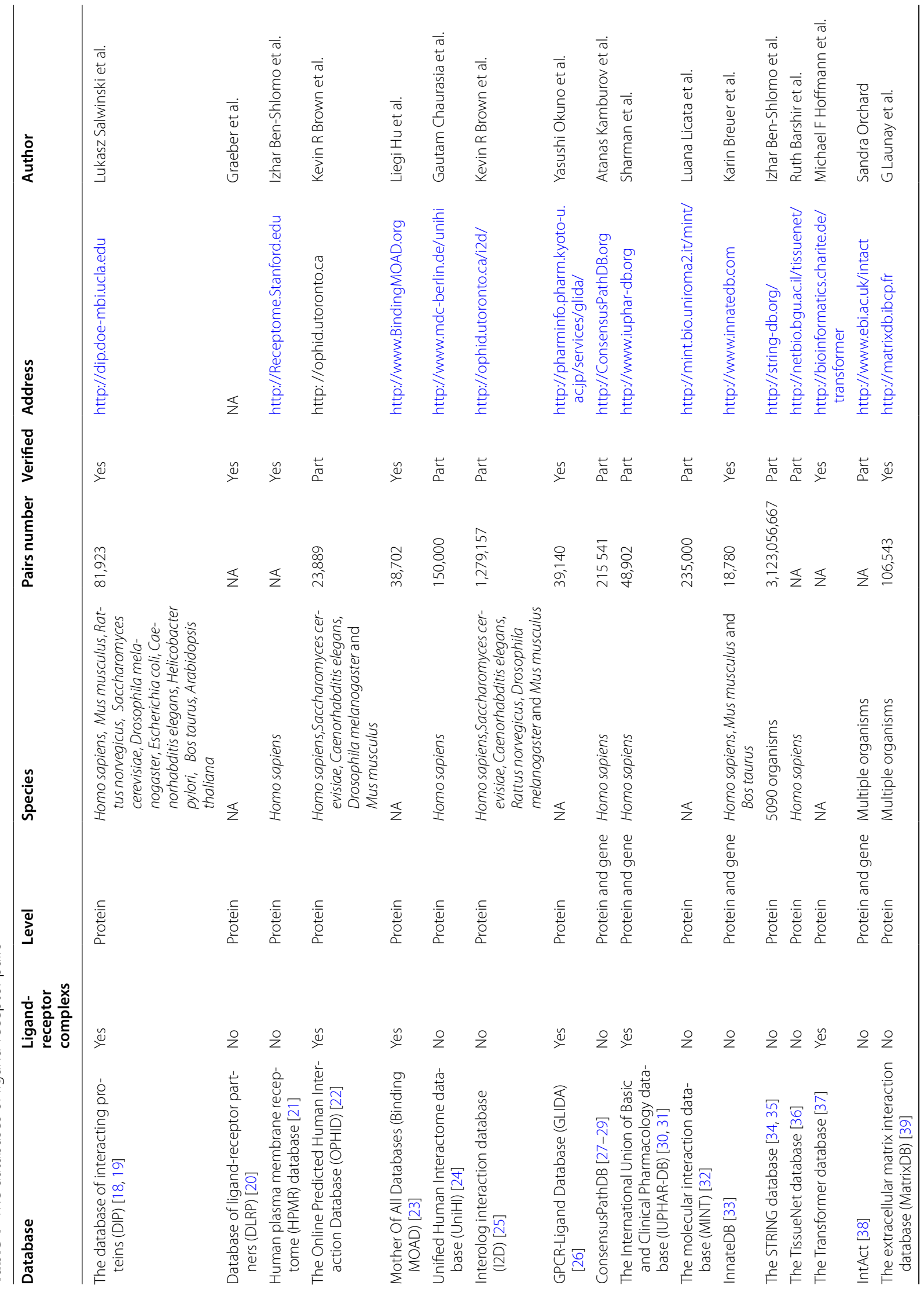




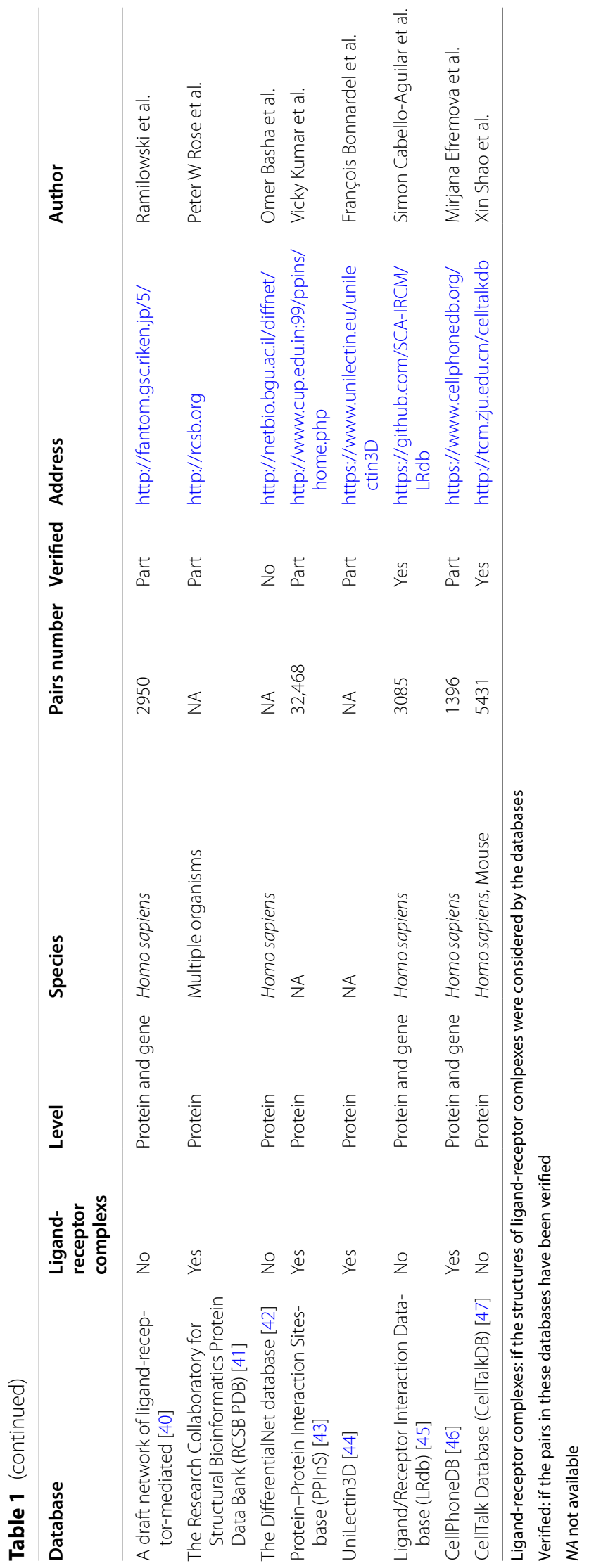




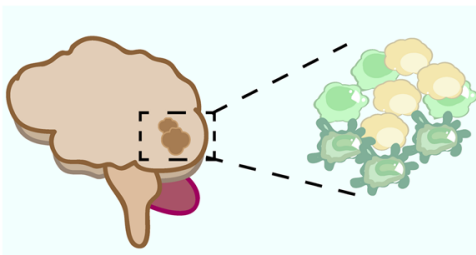

Samples
Dissociation
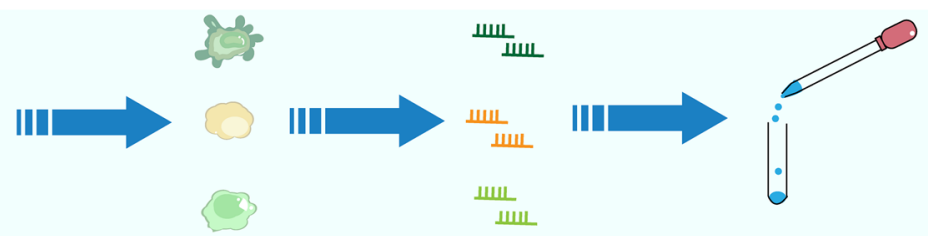

Single cell isolation
RNA extraction and processing
Sequencing analysis

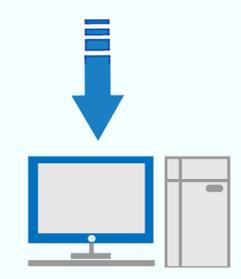

Ligand-receptor analysis
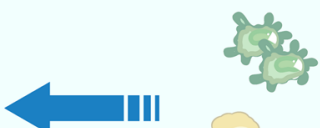

Cell types identification

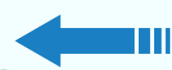

Single cell data analysis

Fig. 1 General procedures of ligand-receptor studies using scRNA-seq techniques. multicellular samples were isolated and captured individual cells. All RNA from each cell was reverse transcribed, amplified and sequenced to obtain transcriptome data for each cell in the sample. Cell types were identified. Then, ligand-receptor interaction could analysis by multiple analysis tools

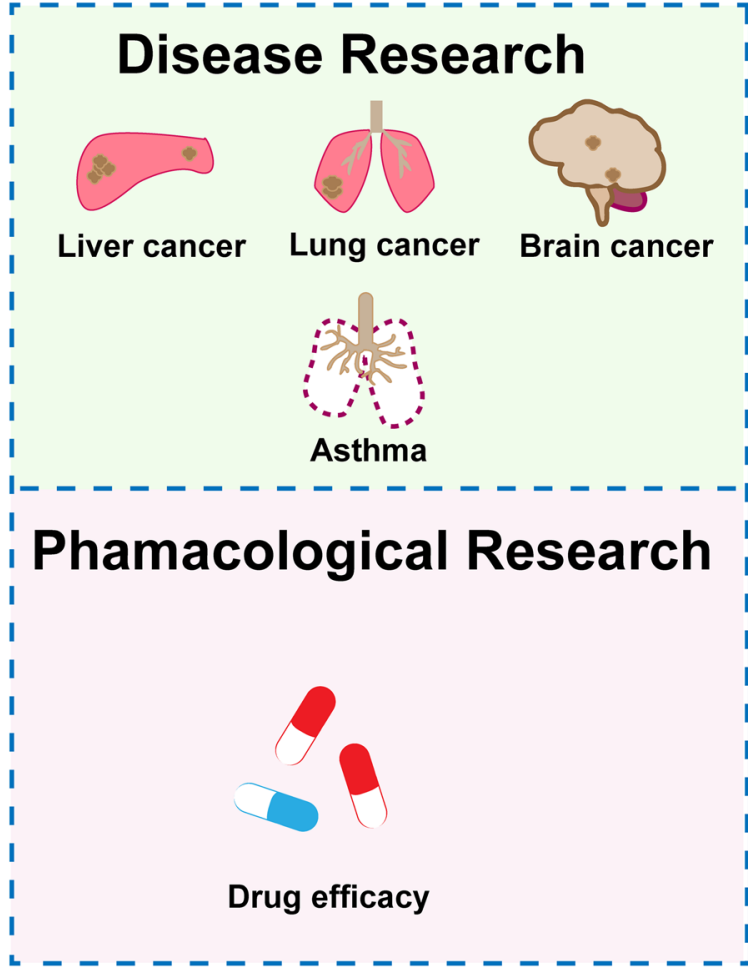

\section{Pathogenic Infection}

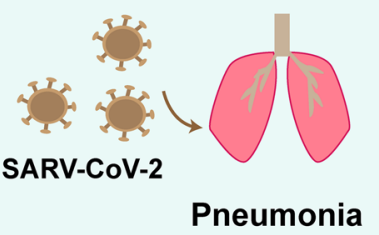

Pneumonia

Physiological Process

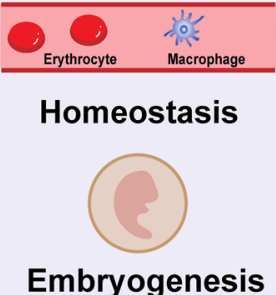

Fig. 2 Current applications of scRNA sequencing in ligand-receptor analysis. The analysis of ligand-receptor interactions using scRNA sequencing can be applicate to elucidate in-depth mechanisms underlying disease research, pathogenic infection, physiological process, pharmacological research 
cells possess the highest number of ligands, while $\mathrm{T}$ cells possess the highest number of receptors, by using scRNA-seq technology: $10 \times$ genomics and Smart-seq2, to analyze cell communications in liver cancer tissues. The main role of dendritic cells in tumor immunity is tumor antigens presentation, migration to lymph nodes, activation of $\mathrm{T}$ cells, etc. [58]. After exploring the intercellular communications between cancer stem-like cells (CSCs) and macrophages in glioma through $10 \times$ genomics, Dongsheng Yuan et al. identified 66 ligand-receptor pairs, some of which could significantly affect prognostic outcomes [59]. Using $10 \times$ genomics, Zhencong Chen et al.. analyzed infiltrating tumor cells, epithelial cells, and $\mathrm{T}$ cells which are identified in the lung adenocarcinoma (LUAD) tumor microenvironment and sequenced by Smart-seq2, built a valid prognostic machine-learning model based on ligand-receptor interactions for predicting the prognosis of LUAD patients [60]. scRNA-seq is used to analyze the communication between tumor cells and immune cells in tumor microenvironment, helping researchers to better analyze tumor development and the body response.

Besides, for other non-neoplastic disease, Braga et al. analyzed changes of ligand-receptor interactions in the airways of healthy individuals and patients with asthma through $10 \times$ genomics. Unbiased analysis of cell-cell interactions identified that the cellular communication network dominated by mesenchymal-epithelial interactions in healthy airways, shifts to type 2 helper $\mathrm{T}$ cells (Th2) dominated interactome in asthmatic lung [61]. Using scRNA-seq to analyze ligand-receptor interactions between cells in diseased tissues can provide insight into the occurrence and progression of the disease.

\section{Pathogenic infection}

Identification of pathogen targeted receptors and targeted cells is the key to treatment of pathogenic infections. Severe Acute Respiratory Syndrome Coronavirus 2 (SARS-CoV-2) has been declared a global public health emergency. Angiotensin I converting enzyme 2 (ACE2) is the host receptor by SARS-CoV-2 to infect human cells. To investigate whether there are other co-receptors/auxiliary proteins as ACE2 partner to facilitate virus entry, Furong Qi et al. explored the single cell gene expression atlas including 119 cell types of 13 human tissues which was mostly sequenced by $10 \times$ genomics, and analyzed the single cell co-expression spectrum of 51 reported RNA virus receptors and 400 other membrane proteins. The result showed that the candidate co-receptors, manifesting the most similar expression patterns with ACE2 across 13 human tissues, are all peptidases, including ANPEP, DPP4 and ENPEP. The crosstalk between coronavirus targets and their surrounding cells indicated that macrophages frequently communicate with the coronavirus targets through chemokine and phagocytosis signaling which highlighting the importance of tissue macrophages in immune defense and immune pathogenesis [62]. Additionally, Qi-Lin Chen et.al. thought that cell receptor-related genes of SARS-CoV-2 is critical for understanding the pathogenesis of SARS-CoV-2 in various tissues, especially in the kidney. Their results showed that ACE2 was widely expressed in specific cell subgroups of various human tissues using 10X genomic, especially in intestinal epithelial cells, kidney proximal tubule (PT) cells, and also alveolar-type (AT) 2 cells of the lung [63]. These results indicate multiple routes for SARS-CoV-2 to infect with human cells/organs and suggest alternative strategies for therapeutic intervention. Studies of ligand-receptor interactions using scRNA-seq provided valuable reference data for the prevention and treatment of current SARS-CoV-2 infection, which are foundation for multi-organ multicellular therapy of pathogen infection.

\section{Physiological process}

The cells of a multicellular organism are derived from a single zygote and genetically identical. Yet, they are phenotypically very different. This difference is the result of a process commonly called cell differentiation [64]. The essence of cell differentiation is the selective expression of intracellular genomes in time and space as cells are stimulated by external signals. Studying the signaling molecules communicated between cells during development is a good way to understand the mechanism of selective cell differentiation during the growth and development of the organism. Roser Vento-Tormo et.al. profiled the transcriptomes and cell-cell communication of about 70,000 single cells using Smart-seq2 and $10 \times$ genomics technology, from first-trimester placentas with matched maternal blood and decidual cells. The results revealed the cellular organization of the decidua and placenta, and the interactions that are critical for placentation and reproductive success [65]. Additionally, Popescu et al. investigated the interaction between erythrocytes and macrophages by Smartseq 2 and $10 \times$ genomics technology. The result showed that some important ligand\&receptor such as VCAM1, ITGB1 and ITGA4, related to hematopoiesis in the fetal hematopoietic system [66]. During the development of the organism, immune cells play an important role in the development of the hematopoietic system.

\section{Pharmacological research}

Moverover, the advent of various targeted drugs which are developed based on ligand-receptor interactions has solved many clinically difficult diseases and improved the 
survival of patients. However, targeted drugs have a narrow range of applicability, and many patients are unable to apply the targeted drugs or have poor therapeutic outcomes. Most current cancer patients do not respond positively to immune checkpoint blockers or have to discontinue their use due to significant side effects. for example, the positive percentage of patients for drugs related to targeting PD-1-PD-L1 rarely exceeds 40\% [67].

Recently, Kathryn E Yost et.al. performed paired single-cell RNA and $\mathrm{T}$ cell receptor sequencing (10X droplet-based sequencing) on 79,046 cells from sitematched tumors from patients with basal or squamous cell carcinoma before and after anti-PD-1 therapy [68]. An increased frequency of follicular helper T cells (Tfh) cells and exhausted/activated CD8 $+\mathrm{T}$ cells appeared after anti-PD-1 treatment, which supporting that PD-1 blockade primarily impacts CD8 $+\mathrm{T}$ cells. Single cell T cell receptor sequencing (scTCR-seq) analysis indicated clonal replacement of exhausted clones when comparing pre- to post-treatment samples, suggesting that $\mathrm{T}$ cell receptor (TCR) dynamics of exhausted cells were mainly influenced by PD-1 blockade, not tumor biopsy timing or location. This may suggest that the effectiveness of targeted drug therapy is closely related to immune cell status. Combination of targeted drugs and immune cell therapy may enhance the therapeutic effect and improve patient survival.

Analysis of ligand-receptor interactions at the single-cell level has shown an important role in science research, helping researchers explore the mechanisms operating on immune cells in the microenvironment in depth. And there is expected to advance disease research and treatment.

\section{Analytical tools for ligand-receptor interactions at single-cell level}

Now, multiple analysis tools for investigating cellular communications through ligand-receptor interactions were developed for deeper analysis of cell crosstalk based on scRNA sequencing (Table 2).

\section{General analysis}

There are many analysis tools designed for cellular communications analysis through ligand-receptor interactions using scRNA-seq. ProximID [69] is an early algorithm for building a cellular network based on physical cell interaction and single-cell mRNA sequencing. It can be used to discover new preferential cellular interactions without prior knowledge of component cell types. And a Tac1+enteroendocrine cell-Lgr5+stem cell interaction in small intestine crypts was identified by ProximID. ProximID is a potent tool for the discovery of new prospective niches, especially when cell types and relative spatial positions are unknown. There's also iTALK [70] (https://github.com/Coolgenome/iTALK). This tool allows to customizate ligand-receptor libraries, and the analysis method is relatively simple and convenient. However, the default analysis species of this tool is human. If you want to analyze other species, there is a need to convert gene to the human corresponding gene.

CellTalker is widely used which was developed by Anthony et al. in 2020 January [71] (https://arc85.github. io/celltalker). This $\mathrm{R}$ package uses a recently described list of receptors and ligands (including soluble ligands such as cytokines) [40] to identify putative ligand-receptor interactions between cell types. And the algorithm is based on differential gene analysis. The ligands/receptors expressions of human papillomavirus (HPV) $\pm \mathrm{CD} 4+\mathrm{T}$ follicular helper cells that is associated with longer progression-free survival in HNSCC patients were significantly different found by cellTalker [71]. Additionally, in 2020 Apr, Teichmann laboratory and Vento-Tormo laboratory co-developed CellPhoneDB [46], which is a python package that's widely used currently. The main advantage of CellPhoneDB over other tools that takes into account the subunit architecture of both ligands and receptors, representing heteromeric complexes accurately. The researchers structured a novel repository of ligands, receptors and their interactions including heteromeric complexes which stores 1086 proteins, 501 are secreted proteins, 585 species as membrane proteins (Table 1). Eric Song et.al. found that in the cerebrospinal fluid (CSF) of COVID-19 patients, these activated innate immune cell populations are predicted to interact with CD8 and CD4 T cells applicating CellPhoneDB, suggesting a coordinated anti-viral immunological response occurring in the CSF of COVID-19 patients [72].

SingleCellSignalR is a new $\mathrm{R}$ package from Simon et al. in June 2020 [45], which used a new regularized product score to assess the confidence in predicted ligand-receptor interactions. It relies on a comprehensive database of known ligand-receptor interactions, which called LRdb (Table 1). LRdb compiled the content of existing databases and integrated informations of Reactome pathways [73] and Gene Ontology Cellular Compartment (GOCC) annotation. Therefore, singleCellSignalR has the abilities to represent a complete intercellular network and to import the latter in systems biology tools such as Cytoscape, and to explore receptor downstream signaling by integrating Reactome and Kyoto Encyclopedia of Genes and Genomes (KEGG) pathways. By mapping mouse genes to their human orthologs according to Ensembl [74] to exploit LRdb, the result of applicating SingleCellSignalR on mouse epidermis data discovered an oriented communication structure from external to basal layers. 


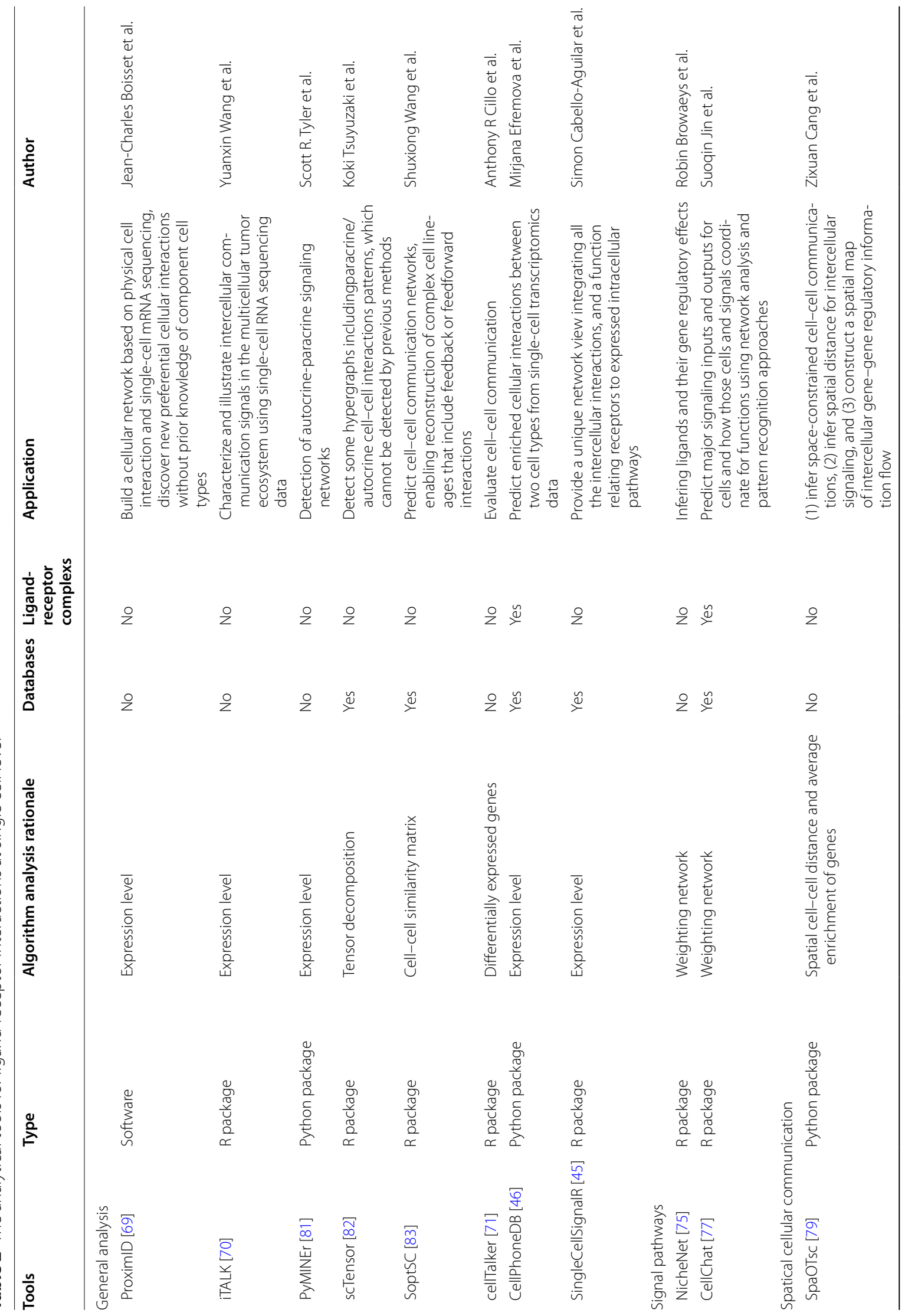




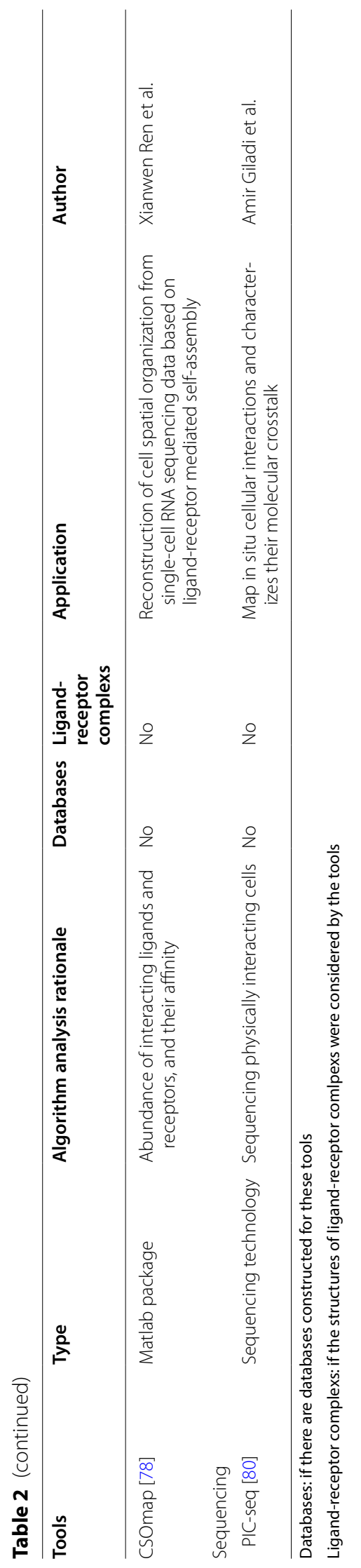




\section{Signal pathways}

In addition to these tools that only anlysis ligand-receptor interactons, there are tools which not only anlysis ligand-receptor interactons, but also explore downstream signal pathways. More recently, Yvan Saeys's team proposed NicheNet [75] (https://github.com/saeyslab/niche netr), which takes into account the changes in the downstream signaling network in the receiver cells, using the estimated downstream signaling changes to predict the activity of the upstream receptors, to identify the functionally influential cell-cell communication. The algorithm is based on a weighting network, which refines the intensity of the interaction and allows infer active ligands and their gene regulatory effects on interacting cells. Andrew L Ji et.al. found a tumor-specific keratinocyte (TSK) population unique to cancer, which localized to a fibrovascular niche. And TSK cells were a hub for intercellular communication revealed by NicheNet [76]. Recently, Suoqin Jin et.al. constructed a database of interactions among ligands, receptors and their cofactors that accurately represents known heteromeric molecular complexes. Based on mass action models, they then developed CellChat, a tool that is able to quantitively infer and analyze intercellular communication networks from scRNA-seq data [77]. Application of CellChat to several scRNA-seq datasets of mouse skin embryonic development and adult wound healing has demonstrated the ability to extract complex signal patterns, including previously known and new ones.

\section{Spatical cellular communications}

Furthermore, Some researcheres believed that cellular communication analysis based on known ligand-receptor interactions could reshape tissue cell structure. CSOmap [78], SpaOTsc [79] are tools that attempt to reconstruct the spatial information of cells through ligand-receptor interactions. CSOmap successfully recapitulate the spatial organization of multiple organs of human and mouse including tumor microenvironments for multiple cancers in pseudo-space, and reveal molecular determinants of cellular interactions [78]. Differently, SpaOTsc not only tries to construct a spatial metric for cells in scRNA-seq data, but also reconstruct cell-cell communication networks by identifying intercellular regulatory relationships between genes.

\section{Sequencing}

In addition to the aforementioned tools for intercellular interaction. do Amit and Amos Tanay jointly presented physically interacting cells sequencing (PIC-seq), which combines cell sorting of physically interacting cells (PICs) with single-cell RNA-sequencing [80]. PIC-seq systematically maps in situ cellular interactions and characterizes their ligand-receptor crosstalk by using computational modeling. Analysis of T cell- dendritic cells pairs reveals an interaction-specific program between pathogen-presenting migratory DCs and $\mathrm{T}$ cells. This method provides a direct and broadly applicable technology to characterize intercellular communication-specific pathways.

For general analysis, expression values of ligand receptor genes are used by many analytical tools to calculate the interaction, unlike cellTalker [71], which uses the differential expression of ligand receptor genes to perform the analysis. SoptSC [83] present similarity matrix-based optimization for single-cell data analysis. And the cellcell relationships learned via the similarity matrix define which cells are clustered. The tools that include signal pathways analysis all use gene weighting networks with different algorithmic scoring points [75, 77]. The two analysis tools that incorporate the spatial localization of cells into the interaction factors differ significantly in their analysis algorithms. SpaOTsc [79] rely on structured optimal transport to recover spatial properties of scRNA-seq data by utilizing spatial measurements of a relatively small number of genes. SpaOTsc has broader applications, both in integrating non-spatial single-cell measurements with spatial data, and directly in spatial single-cell transcriptomics data to reconstruct spatial cellular dynamics in tissues. For CSOmap [78], the algorithmic process is composed of two main steps. The first is to estimate the cellular interacting potentials by integrating thousands of ligand-receptor pairs, resulting in a cell-bycell affinity matrix. The second is to embed the inherently high-dimensional affinity matrix into three-dimensional space.

For these four classes of analysis tools, general analysis and signal pathways are more widely applicable and almost all scRNA-seq datas can be used for analysis. Spatical cellular communication analysis is suitable for analyzing tissue scRNA-seq datas, and the technology is not fully mature yet and needs to be combined with the results of wet experiments. Sequencing method which refer to PIC-seq, is suitable for dissecting cellular crosstalk of physically interacting cells, and could characterize intercellular interaction-specific pathways at high resolution. PIC-seq is a direct and broadly applicable technology [80].

The tools for ligand-receptor interaction at single cell level are collated in Table 2. These analytical tools and methods greatly facilitate researchers in quantifying the statistical significance of cell-cell interactions and reveal the potentially critical ligand-receptor pairs mediating such interactions. It will gain new insights into the role of cells. 


\section{Inadequacy of current research}

Although many tools have been developed and applied extensively in the study of receptors and ligands, there are still limitations of these tools. One problem with all the tools is the reliance on databases of known ligandreceptor pairs interactions. However, continuous update of current ligand-receptor libraries is necessary to cover more information of receptors, ligands and their interactions. Recently, receptor study of immunoglobulin superfamily (IGSFF) identified more than 60 new pairs of ligand -receptor [84]. The ligand-receptor pairs network is not completely understood and still needs futher exploration and improvement. Secondly, there is still a lack of understanding of ligand-receptor binding complexes at protein level, which the actual interactions occur, since most of the current studies of cellular interactions focus on genetic analysis. Post-transcriptional modification, binding mode, and the affinity strength etc. are yet to be thoroughly learnt. The integrated data which integrated the transcriptome profile with highly multiplexed proteomic and genomic data, was more informative than transcriptome data alone [85]. Thirdly, spatial location of tissue cells is vital. Spatiotemporal alterations in the microenvironment have a considerable impact on cells interactions. However, current single-cell sequencing technology cannot survey the specific spatial locations of cells. Lastly, the real cellular communication is carried out with single cell as a unit, which current researches are analyzed with cell types.

With the recognition of the complexity of the disease, personalized precision treatment is the core of treatment. Study of ligand-receptor pairs interactions using single-cell sequencing technology unveils the complex cell communication networks. Cellular communication is a very complicated process which is achieve through ligand-receptor signaling, and other mechanisms including pressure stimulation, concentration regulation, and intracellular signal transduction. Further extensive study is needed to get a deeper insight into these inter- and intra-cellular interactions for better understanding of disease progression and discovery of potential drug targets.

\section{Conclusion}

Cell-cell communication governs the biological behaviors of multicellular populations. Ligand-receptor interactions, which is a vital type of cellular communication, have presented important roles in pharmacological research and disease progression as reviewed. Now, the emergence of scRNA sequencing technology gives a new way that are closer to the actual action of organisms for the research of ligand-receptor interaction. Many studies have learning cellular communication based on ligand-receptor interaction by scRNA sequencing (Fig. 2), to identify ligand-receptor interactions as biomarkers or potential therapeutic targets. Thus, tools or methods for learning ligand-receptor interactions by scRNA sequencing were developed, and sorted out in this study. It is expectant that us these tools to study diseases in more depth.

\section{Abbreviations \\ scRNA-seq: Single-cell RNA sequencing; FH: Familial Hypercholesterolemia; LDLR: Low density lipoprotein receptor; CTLA-4: Cytotoxic T lymphocyte-asso- ciated antigen-4; PD-1: Programmed death 1; ICOS: Inducible co-stimulator; TIM-3: T cell immunoglobulin and mucin domain 3; DLRP: Ligand-receptor pairing database; IUPHAR: International Union of Basic and Clinical Pharmacol- ogy; HPMR: Human plasma membrane receptor group; HPRD: Human Protein Reference Database; UniHI: Unified Human Interactome database; CellTalkDB: CellTalk database; MOAD: Mother of All Databases; GPCRs: G-protein coupled receptors; GLIDA: G protein coupled ligand-receptor pairs database; UCSF: University of California; CSCs: Cancer stem-like cells; LUAD: Lung adenocarci- noma; SARS-CoV-2: Severe Acute Respiratory Syndrome Coronavirus 2; ACE2: Angiotensin I converting enzyme 2; PT: Proximal tubule cells; Tfh: Follicular helper T cells; scTCR-seq: Single cell T cell receptor sequencing; TCR: T cell receptor; CSF: Cerebrospinal fluid; GOCC: Gene Ontology Cellular Compart- ment; KEGG: Kyoto Encyclopedia of Genes and Genomes; TSK:Tumor-specific keratinocyte; PIC-seq: Physically interacting cells sequencing; PICs: Physically interacting cells; IGSFF: Immunoglobulin superfamily.}

\section{Acknowledgements}

Not applicable.

\section{Authors' contributions}

FM wrote and revised the manuscript and tables; SWZ generated all figures and provided some suggestions; BZW provided some suggestions and helped to review manuscript. LHS provided some suggestions and expanded the manuscript; LLW and FMZ revised both text and figures critically for important intellectual content; all four authors have given final approval of the version to be published. All authors read and approved the final manuscript.

\section{Funding}

This study was supported by National Natural Science Foundation of China (NO.81773267, NO.81672670).

Availability of data and materials

Not applicable.

\section{Declarations}

Ethics approval and consent to participate

Not applicable.

Consent for publication

Not applicable.

Competing interests

The authors declare that they have no competing interests.

\section{Author details}

${ }^{1}$ Department of Microbiology, Harbin Medical University, Harbin 150081, China. ${ }^{2}$ Wu Lien-Teh Institute, Harbin Medical University, Harbin 150081, China. ${ }^{3}$ Shenzhen Third People's Hospital, Second Hospital, Affiliated to Southern University of Science and Technology, Shenzhen 518112, China. 
Received: 30 November 2020 Accepted: 22 June 2021

Published online: 03 July 2021

\section{References}

1. Singer SJ. Intercellular communication and cell-cell adhesion. Science. 1992;255:1671-7.

2. Wang X, Song W, Kawazoe N, Chen G. The osteogenic differentiation of mesenchymal stem cells by controlled cell-cell interaction on micropatterned surfaces. J Biomed Mater Res A. 2013;101:3388-95.

3. Shao X, Lu X, Liao J, Chen H, Fan X. New avenues for systematically inferring cell-cell communication: through single-cell transcriptomics data. Protein Cell. 2020. https://doi.org/10.1007/s13238-020-00727-5.

4. Finlay DB, Duffull SB, Glass M. 100 years of modelling ligandreceptor binding and response: A focus on GPCRs. Br J Pharmacol. 2020;177:1472-84.

5. Chen H, Ye F, Guo G. Revolutionizing immunology with single-cell RNA sequencing. Cell Mol Immunol. 2019;16:242-9.

6. Hao S, Yan KK, Ding L, Qian C, Chi H, Yu J. Network approaches for dissecting the immune system. Science. 2020;23:101354.

7. Olsnes S, Pihl A. Clinical significance of estrogen receptors in human breast cancer. Biomedicine. 1974;20:377-83.

8. Lee J, Pilch PF. The insulin receptor: structure, function, and signaling. Am J Physiol. 1994;266:C319-334.

9. Defesche JC, Gidding SS, Harada-Shiba M, Hegele RA, Santos RD, Wierzbicki AS. Familial hypercholesterolaemia. Nat Rev Dis Primers. 2017;3:17093.

10. Leach DR, Krummel MF, Allison JP. Enhancement of antitumor immunity by CTLA-4 blockade. Science. 1996;271:1734-6.

11. Chen DS, Mellman I. Oncology meets immunology: the cancer-immunity cycle. Immunity. 2013;39:1-10.

12. O'Donnell JS, Teng MWL, Smyth MJ. Cancer immunoediting and resistance to T cell-based immunotherapy. Nat Rev Clin Oncol. 2019;16:151-67.

13. Kong X. Discovery of new immune checkpoints: family grows up. Adv Exp Med Biol. 2020;1248:61-82.

14. Lang PT, Brozell SR, Mukherjee S, Pettersen EF, Meng EC, Thomas V, Rizzo RC, Case DA, James TL, Kuntz ID. DOCK 6: combining techniques to model RNA-small molecule complexes. RNA. 2009;15:1219-30.

15. Goodsell DS, Morris GM, Olson AJ. Automated docking of flexible ligands: applications of AutoDock. J Mol Recognit. 1996;9:1-5.

16. Morris GM, Huey R, Lindstrom W, Sanner MF, Belew RK, Goodsell DS, Olson AJ. AutoDock4 and AutoDockTools4: Automated docking with selective receptor flexibility. J Comput Chem. 2009;30:2785-91.

17. Davis IW, Baker D. RosettaLigand docking with full ligand and receptor flexibility. J Mol Biol. 2009;385:381-92.

18. Xenarios I, Rice DW, Salwinski L, Baron MK, Marcotte EM, Eisenberg D. DIP: the database of interacting proteins. Nucleic Acids Res. 2000;28:289-91.

19. Salwinski L, Miller CS, Smith AJ, Pettit FK, Bowie JU, Eisenberg D. The database of interacting proteins: 2004 update. Nucleic Acids Res. 2004;32:D449-451.

20. Graeber TG, Eisenberg D. Bioinformatic identification of potential autocrine signaling loops in cancers from gene expression profiles. Nat Genet. 2001;29:295-300.

21. Ben-Shlomo I, Yu Hsu S, Rauch R, Kowalski HW, Hsueh AJ. Signaling receptome: a genomic and evolutionary perspective of plasma membrane receptors involved in signal transduction. Sci STKE. 2003. 2003:RE9.

22. Brown KR, Jurisica I. Online predicted human interaction database. Bioinformatics. 2005;21:2076-82.

23. Hu L, Benson ML, Smith RD, Lerner MG, Carlson HA. Binding MOAD (Mother Of All Databases). Proteins. 2005;60:333-40.

24. Chaurasia G, Iqbal Y, Hanig C, Herzel H, Wanker EE, Futschik ME. UniHI: an entry gate to the human protein interactome. Nucleic Acids Res. 2007;35:D590-594.

25. Brown KR, Jurisica I. Unequal evolutionary conservation of human protein interactions in interologous networks. Genome Biol. 2007;8:R95.

26. Okuno Y, Tamon A, Yabuuchi H, Niijima S, Minowa Y, Tonomura K, Kunimoto R, Feng C. GLIDA: GPCR-ligand database for chemical genomics drug discovery-database and tools update. Nucleic Acids Res. 2008:36:D907-912.
27. Kamburov A, Wierling C, Lehrach $\mathrm{H}$, Herwig R. ConsensusPathDB-a database for integrating human functional interaction networks. Nucleic Acids Res. 2009:37:D623-628.

28. Kamburov A, Pentchev K, Galicka H, Wierling C, Lehrach H, Herwig R. ConsensusPathDB: toward a more complete picture of cell biology. Nucleic Acids Res. 2011;39:D712-717.

29. Kamburov A, Stelzl U, Lehrach H, Herwig R. The ConsensusPathDB interaction database: 2013 update. Nucleic Acids Res. 2013;41:D793-800.

30. Sharman JL, Mpamhanga CP, Spedding M, Germain P, Staels B, Dacquet C, Laudet V, Harmar AJ, Nc I. IUPHAR-DB: new receptors and tools for easy searching and visualization of pharmacological data. Nucleic Acids Res. 2011;39:D534-538.

31. Sharman JL, Benson HE, Pawson AJ, Lukito V, Mpamhanga CP, Bombail V, Davenport AP, Peters JA, Spedding M, Harmar AJ, Nc I. IUPHARDB: updated database content and new features. Nucleic Acids Res. 2013:41:D1083-1088

32. Licata L, Briganti L, Peluso D, Perfetto L, lannuccelli M, Galeota E, Sacco F, Palma A, Nardozza AP, Santonico E, et al. MINT, the molecular interaction database: 2012 update. Nucleic Acids Res. 2012;40:D857-861.

33. Breuer K, Foroushani AK, Laird MR, Chen C, Sribnaia A, Lo R, Winsor GL Hancock RE, Brinkman FS, Lynn DJ. InnateDB: systems biology of innate immunity and beyond-recent updates and continuing curation. Nucleic Acids Res. 2013;41:D1228-1233.

34. Franceschini A, Szklarczyk D, Frankild S, Kuhn M, Simonovic M, Roth A, Lin J, Minguez P, Bork P, von Mering C, Jensen LJ. STRING v9.1: protein-protein interaction networks, with increased coverage and integration. Nucleic Acids Res. 2013:41:D808-815.

35. Szklarczyk D, Franceschini A, Wyder S, Forslund K, Heller D, Huerta-Cepas I, Simonovic M, Roth A, Santos A, Tsafou KP, et al. STRING v10: protein-protein interaction networks, integrated over the tree of life. Nucleic Acids Res. 2015:43:D447-452.

36. Barshir R, Basha O, Eluk A, Smoly IY, Lan A, Yeger-Lotem E. The TissueNet database of human tissue protein-protein interactions. Nucleic Acids Res. 2013:41:D841-844.

37. Hoffmann MF, Preissner SC, Nickel J, Dunkel M, Preissner R, Preissner S. The Transformer database: biotransformation of xenobiotics. Nucleic Acids Res. 2014;42:D1113-1117.

38. Orchard S, Ammari M, Aranda B, Breuza L, Briganti L, BroackesCarter F, Campbell NH, Chavali G, Chen C, delToro N, et al. The MIntAct projectIntAct as a common curation platform for 11 molecular interaction databases. Nucleic Acids Res. 2014;42:358-63.

39. Launay G, Salza R, Multedo D, Thierry-Mieg N, Ricard-Blum S. MatrixDB, the extracellular matrix interaction database: updated content, a new navigator and expanded functionalities. Nucleic Acids Res. 2015;43:D321-327.

40. Ramilowski JA, Goldberg T, Harshbarger J, Kloppmann E, Lizio M, Satagopam VP, Itoh M, Kawaji H, Carninci P, Rost B, Forrest AR. A draft network of ligand-receptor-mediated multicellular signalling in human. Nat Commun. 2015;6:7866.

41. Rose PW, Prlic A, Altunkaya A, Bi C, Bradley AR, Christie CH, Costanzo LD, Duarte JM, Dutta S, Feng Z, et al. The RCSB protein data bank: integrative view of protein, gene and 3D structural information. Nucleic Acids Res. 2017:45:D271-81.

42. Basha O, Shpringer R, Argov CM, Yeger-Lotem E. The DifferentialNet database of differential protein-protein interactions in human tissues. Nucleic Acids Res. 2018;46:D522-6.

43. Kumar V, Mahato S, Munshi A, Kulharia M. PPInS: a repository of proteinprotein interaction sitesbase. Sci Rep. 2018;8:12453.

44. Bonnardel F, Mariethoz J, Salentin S, Robin X, Schroeder M, Perez S, Lisacek F, Imberty A. UniLectin3D, a database of carbohydrate binding proteins with curated information on 3D structures and interacting ligands. Nucleic Acids Res. 2019;47:D1236-44.

45. Cabello-Aguilar S, Alame M, Kon-Sun-Tack F, Fau C, Lacroix M, Colinge J. SingleCellSignalR: inference of intercellular networks from single-cell transcriptomics. Nucleic Acids Res. 2020;48:e55.

46. Efremova M, Vento-Tormo M, Teichmann SA, Vento-Tormo R. Cell PhoneDB: inferring cell-cell communication from combined expression of multi-subunit ligand-receptor complexes. Nat Protoc. 2020;15:1484-506

47. XinShao JL, Chengyu L, Xiaohui F. Cell TalkDB. Brief Bioinform. 2020. https://doi.org/10.1093/bib/bbaa269. 
48. Cai L, Friedman N, Xie XS. Stochastic protein expression in individual cells at the single molecule level. Nature. 2006;440:358-62.

49. Rosenfeld N, Young JW, Alon U, Swain PS, Elowitz MB. Gene regulation at the single-cell level. Science. 2005;307:1962-5.

50. Garalde DR, Snell EA, Jachimowicz D, Sipos B, Lloyd JH, Bruce M, Pantic N, Admassu T, James P, Warland A, et al. Highly parallel direct RNA sequencing on an array of nanopores. Nat Methods. 2018;15:201-6.

51. van Dijk EL, Jaszczyszyn $Y$, Naquin $D$, Thermes $C$. The third revolution in sequencing technology. Trends Genet. 2018;34:666-81.

52. Chappell L, Russell AJC, Voet T. Single-Cell (Multi)omics Technologies. Annu Rev Genomics Hum Genet. 2018;19:15-41.

53. Tang F, Barbacioru C, Wang Y, Nordman E, Lee C, Xu N, Wang X, Bodeau J, Tuch BB, Siddiqui A, et al. mRNA-Seq whole-transcriptome analysis of a single cell. Nat Methods. 2009;6:377-82.

54. Stark R, Grzelak M, Hadfield J. RNA sequencing: the teenage years. Nat Rev Genet. 2019:20:631-56.

55. Regev A, Teichmann SA, Lander ES, Amit I, Benoist C, Birney E, Bodenmiller B, Campbell P, Carninci P, Clatworthy M, et al. The human cell Atlas. elife. 2017;6:e27041.

56. Insel TR, Landis SC, Collins FS. Research priorities The NIH BRAIN Initiative. Science. 2013;340:687-8.

57. Hedlund E, Deng Q. Single-cell RNA sequencing: Technical advancements and biological applications. Mol Aspects Med. 2018;59:36-46.

58. Zhang Q, He Y, Luo N, Patel SJ, Han Y, Gao R, Modak M, Carotta S, Haslinger C, Kind D, et al. Landscape and dynamics of single immune cells in hepatocellular carcinoma. Cell. 2019;179:829-845820.

59. Yuan D, Tao Y, Chen G, Shi T. Systematic expression analysis of ligandreceptor pairs reveals important cell-to-cell interactions inside glioma. Cell Commun Signal. 2019;17:48

60. Chen Z, Yang X, Bi G, Liang J, Hu Z, Zhao M, Li M, Lu T, Zheng Y, Sui Q, et al. Ligand-receptor interaction atlas within and between tumor cells and $\mathrm{T}$ cells in lung adenocarcinoma. Int J Biol Sci. 2020;16:2205-19.

61. Vieira Braga FA, Kar G, Berg M, Carpaij OA, Polanski K, Simon LM, Brouwer S, Gomes T, Hesse L, Jiang J, et al. A cellular census of human lungs identifies novel cell states in health and in asthma. Nat Med. 2019;25:1153-63.

62. Qi F, Qian S, Zhang S, Zhang Z. Single cell RNA sequencing of 13 human tissues identify cell types and receptors of human coronaviruses. Biochem Biophys Res Commun. 2020;526:135-40.

63. Chen QL, Li JQ, Xiang ZD, Lang Y, Guo GJ, Liu ZH. Localization of cell receptor-related genes of SARS-CoV-2 in the Kidney through single-cell transcriptome analysis. Kidney Dis (Basel). 2020;6:258-70.

64. Paldi A. Conceptual challenges of the systemic approach in understanding cell differentiation. Methods Mol Biol. 2018;1702:27-39.

65. Vento-Tormo R, Efremova M, Botting RA, Turco MY, Vento-Tormo M, Meyer KB, Park JE, Stephenson E, Polanski K, Goncalves A, et al. Single-cell reconstruction of the early maternal-fetal interface in humans. Nature. 2018;563:347-53

66. Popescu DM, Botting RA, Stephenson E, Green K, Webb S, Jardine L, Calderbank EF, Polanski K, Goh I, Efremova M, et al. Decoding human fetal liver haematopoiesis. Nature. 2019;574:365-71.

67. Rizvi NA, Hellmann MD, Snyder A, Kvistborg P, Makarov V, Havel JJ, Lee W, Yuan J, Wong P, Ho TS, et al. Cancer immunology Mutational landscape determines sensitivity to PD-1 blockade in non-small cell lung cancer. Science. 2015;348:124-8.

68. Yost KE, Satpathy AT, Wells DK, Qi Y, Wang C, Kageyama R, McNamara KL, Granja JM, Sarin KY, Brown RA, et al. Clonal replacement of tumor-specific T cells following PD-1 blockade. Nat Med. 2019;25:1251-9.

69. Boisset JC, Vivie J, Grun D, Muraro MJ, Lyubimova A, van Oudenaarden A. Mapping the physical network of cellular interactions. Nat Methods. 2018:15:547-53
70. Wang Y, Wang R, Zhang S, Song S, Jiang C, Han G, Wang M, Ajani J, Futreal A, Wang L. iTALK: an R package to characterize and illustrate intercellular communication. BioRxiv. 2019. https://doi.org/10.1101/507871.

71. Cillo AR, Kurten CHL, Tabib T, Qi Z, Onkar S, Wang T, Liu A, Duvvuri U, Kim $\mathrm{S}$, Soose RJ, et al. Immune landscape of viral- and carcinogen-driven head and neck cancer. Immunity. 2020;52:183-199 e189.

72. Song E, Chow RD, Jiang R, Zamecnik CR, Loudermilk RP, Dai Y, Liu F, Geng $B$, Chiarella J, Israelow B, et al. Immunologically distinct responses occur in the CNS of COVID-19 patients. bioRxiv. 2020. https://doi.org/10.1101/ 2020.09.11.293464.

73. Fabregat A, Jupe S, Matthews L, Sidiropoulos K, Gillespie M, Garapati P, Haw R, Jassal B, Korninger F, May B, et al. The reactome pathway knowledgebase. Nucleic Acids Res. 2018;46:D649-55.

74. Flicek P, Aken BL, Beal K, Ballester B, Caccamo M, Chen Y, Clarke L, Coates G, Cunningham F, Cutts T, et al. Ensembl 2008. Nucleic Acids Res. 2008:36:D707-714

75. Browaeys R, Saelens W, Saeys Y. NicheNet: modeling intercellular communication by linking ligands to target genes. Nat Methods. 2020;17:159-62.

76. Ji AL, Rubin AJ, Thrane K, Jiang S, Reynolds DL, Meyers RM, Guo MG, George BM, Mollbrink A, Bergenstrahle J, et al. Multimodal analysis of composition and spatial architecture in human squamous cell carcinoma. Cell. 2020;182:497-514 e422.

77. Jin S, Juarez C, Zhang L, Chang I, Myung P, Plikus M, Nie Q. Inference and analysis of cell-cell communication using Cell Chat. BioRxiv. 2020. https:// doi.org/10.1101/2020.07.21.214387.

78. Ren X, Zhong G, Zhang Q, Zhang L, Sun Y, Zhang Z. Reconstruction of cell spatial organization from single-cell RNA sequencing data based on ligand-receptor mediated self-assembly. Cell Res. 2020;30:763-78.

79. Cang Z, Nie Q. Inferring spatial and signaling relationships between cells from single cell transcriptomic data. Nat Commun. 2020;11:2084.

80. Giladi A, Cohen M, Medaglia C, Baran Y, Li B, Zada M, Bost P, BlecherGonen R, Salame TM, Mayer JU, et al. Dissecting cellular crosstalk by sequencing physically interacting cells. Nat Biotechnol. 2020;38:629-37.

81. Tyler SR, Rotti PG, Sun X, Yi Y, Xie W, Winter MC, Flamme-Wiese MJ, Tucker BA, Mullins RF, Norris AW, Engelhardt JF. PyMINEr finds gene and autocrine-paracrine networks from human islet scRNA-Seq. Cell Rep. 2019;26:1951-1964 e1958.

82. Tsuyuzaki K, Ishii M, Nikaido I. Uncovering hypergraphs of cell-cell interaction from single cell RNA-sequencing data. BioRxiv. 2019. https://doi.org/ $10.1101 / 566182$.

83. Wang S, Karikomi M, MacLean AL, Nie Q. Cell lineage and communication network inference via optimization for single-cell transcriptomics. Nucleic Acids Res. 2019;47:e66.

84. Verschueren E, Husain B, Yuen K, Sun Y, Paduchuri S, Senbabaoglu Y, Lehoux I, Arena TA, Wilson B, Lianoglou S, et al. The immunoglobulin superfamily receptome defines cancer-relevant networks associated with clinical outcome. Cell. 2020;182:329-344 e319.

85. Choi JR, Yong KW, Choi JY, Cowie AC. Single-cell RNA sequencing and its combination with protein and DNA analyses. Cells. 2020;9:1130.

\section{Publisher's Note}

Springer Nature remains neutral with regard to jurisdictional claims in published maps and institutional affiliations.

Ready to submit your research? Choose BMC and benefit from:

- fast, convenient online submission

- thorough peer review by experienced researchers in your field

- rapid publication on acceptance

- support for research data, including large and complex data types

- gold Open Access which fosters wider collaboration and increased citations

- maximum visibility for your research: over 100M website views per year

At BMC, research is always in progress.

Learn more biomedcentral.com/submissions 